\title{
A formação em serviço social para a intervenção na diversidade de género e sexual
}

\author{
Social work training for intervention in gender and sexual diversity
}

Nélson Ramalho ${ }^{1}$

\begin{abstract}
Resumo
As organizações nacionais e internacionais de serviço social têm vindo a assumir o compromisso de proteção e preservação dos direitos das pessoas LGBTI por reconhecerem as múltiplas opressões a que estão sujeitas. Todavia, as escolas de serviço social continuam a fornecer pouca formação sobre género e sexualidade, o que contribui para os assistentes sociais não estarem qualificados para lidar e responder adequadamente às necessidades das pessoas LGBTI. O presente artigo fundamenta, assim, a necessidade das escolas de serviço social promoverem conhecimentos e desenvolverem competências específicas para a intervenção com populações com identidades de género e/ou orientações sexuais diversas, e apresenta recomendações que poderão contribuir para esse trabalho pedagógico de formação e qualificação profissional.
\end{abstract}

Palavras-chave: LGBTI, género, sexualidade, formação, serviço social.

\begin{abstract}
National and international social service associations have been committed to protecting and preserving the rights of LGBTI people as they recognize the multiple oppressions they are related to. However, as schools of social service you need to provide training equipment on gender and sexuality, which contributes to the fact that social workers are not assigned to deal with and respond to the needs of LGBTI people. The present article therefore supports the need for social service schools to promote knowledge and develop specific skills for intervention with categories with gender identities and / or different sexual orientations, and present specifications that contribute to this educational training and professional qualification work.
\end{abstract}

Key words: LGBTI, gender, sexuality, training, social work.

\section{Introdução}

O presente artigo discute a necessidade dos assistentes sociais estarem qualificados profissionalmente para intervir com pessoas Lésbicas, Gays, Bissexuais, Transgénero e Intersexo (LGBTI), ressaltando o papel das escolas de serviço social na disponibilização de formação adequada sobre género e sexualidade. Para tal, serão apresentados, num primeiro momento, as exigências deontológicas e os compromissos de proteção e preservação dos direitos das pessoas LGBTI assumidos pelas organizações nacionais e

\footnotetext{
1 Professor Auxiliar do Instituto de Serviço Social da Universidade Lusófona | ISCTE - Instituto Universitário de Lisboa (ISCTE-IUL), Centro de Investigação e Estudos de Sociologia, Lisboa, Portugal | E-mail: nelson.ramalho@ulusofona.pt
} 
internacionais de serviço social, num segundo momento, pesquisas empíricas que evidenciam que os assistentes sociais continuam a apresentar baixos conhecimentos e competências para intervir com populações com identidades de género e /ou orientações sexuais diversas e, por fim, num terceiro momento, algumas recomendações que poderão contribuir para o trabalho pedagógico de formação e qualificação profissional.

\section{Proteção e Preservação dos Direitos LGBTI: Os Compromissos Assumidos}

A diversidade é uma componente da realidade social, pelo que as particularidades que unem e diferenciam os grupos ou categorias sociais não deveriam resultar em discriminação, desigualdade e/ou exclusão. Quando tal acontece é porque as diferenças não são socialmente aceites e, desse modo, a luta pelo reconhecimento do "direito à diferença" - uma das dimensões dos direitos humanos - acaba por implicar questões éticas e políticas (Barroco, 2003). O serviço social, enquanto profissão de direitos humanos, obriga os seus profissionais, através do código deontológico (Associação dos Profissionais de Serviço Social, 2018), a "respeitar incondicionalmente o valor de cada pessoa e a sua integridade, reconhecendo a diversidade" (alínea a do ponto 8.1.), a "ser intolerantes com a discriminação negativa" (alínea b do ponto 8.1) e a "contribuir para a redução de todas as formas de opressão" (alínea a do ponto 8.3), nas quais se incluem as opressões baseadas na identidade de género e/ou orientação sexual. Aos assistentes sociais compete, pois, acolher a diversidade de pessoas LGBTI, explorar os preconceitos que possam resultar num tratamento discriminatório e desigual em termos de respeito, direitos, recursos ou oportunidades e apoiar todas aquelas que são vulneráveis à opressão.

Reconhecendo que a discriminação é prejudicial para o bem-estar social, emocional, psicológico, físico e económico das pessoas LGBTI (Ramalho, 2015), a International Federation of Social Workers (IFSW) declarou, em 2014, na Conferência Mundial de Serviço Social, realizada em Melbourne (Austrália), a necessidade da profissão se envolver ativamente na "proteção e preservação dos direitos das pessoas LGBTI" (ponto 5.1.) na medida em que eles se constituem direitos humanos. Acreditando "que as políticas e programas destinados a promover o bem-estar" da sociedade "não serão bem-sucedidas se não for prestada atenção à discriminação contra as pessoas LGBTI" (ponto 5.5), a ISFW e as suas organizações membros assumiram o compromisso de "trabalhar para promover o desenvolvimento de políticas, implementar programas e ação social para melhorar o bem-estar das pessoas LGBTI de todas as idades” (ponto 5.3). Tal compromisso exige, porém, que o serviço social adote práticas anti discriminatórias 
e anti opressivas (Brown e Cocker, 2011; Dominelli, 1998; Okitikpi e Aymer, 2010), orientadas para uma luta política (Baines, 2007) capaz de questionar os sistemas estruturais geradores de desigualdades que alimentam o heterossexismo, a homo/transfobia ou o sexismo; de alterar as relações sociais de poder existentes, desde o nível macro ao nível micro; de incentivar o respeito pelas diferenças individuais e/ou grupais; e de celebrar a pluralidade identitária.

\section{O Problema da Desqualificação Profissional}

Apesar das exigências deontológicas e dos compromissos assumidos pelas organizações nacionais e internacionais de serviço social relacionados com a necessidade de proteção e preservação dos direitos LGBTI, a investigação científica tem evidenciado que grande parte dos profissionais tem pouco ou nenhum conhecimento sobre as questões da diversidade de género (Erich et al., 2007; Mallon, 1999a e Pyne, 2016) ou sexual (Hicks, 2008; Jeyasingham, 2008; Morton, Jeyasingham e Hicks, 2013; Radecki, 2011; Rocha, 2009), além de que a sua experiência profissional nestes campos é "muito limitada" (Mallon, 1999a, p. 4). Com efeito, os assistentes sociais acabam por não estar preparados para lidar e responder adequadamente às necessidades das pessoas LGBTI (Charnley e Langley, 2007; Cohen, Padilla e Aravena, 2006; Mallon. 1999a), situação que é espelhada nos deficitários recursos dirigidos a elas e no desenvolvimento de serviços sociais predominantemente cis/heteronormativos. Tal ocorre porque, em parte, as escolas de serviço social tendem a fornecer pouca formação e literatura profissional sobre identidade de género e orientação sexual (Bergh e Crisp, 2004; Erich et al., 2007; Mackelprang, Ray e Hernandez-Peck, 1996; Mallon, 1999a,b; McPhail, 2008; Rocha, 2009), a promover incipientes oportunidades de estágios curriculares com populações LGBTI (Messinger, 2004; Newman, Bogo e Daley, 2009) e a produzir um baixo número de investigações científicas focadas na diversidade de género e sexual (Mallon, 1999a; Rocha, 2009). Deste modo, parece não ter havido, ainda, um esforço para se integrar e explorar, com maior intensidade, estas temáticas nos planos de estudo dos diferentes ciclos académicos de serviço social.

Modelos de formação não questionados podem contribuir para a perpetuação da discriminação. À semelhança do trabalho realizado por Maria Gabriela Moita (2001), que observou a existência de níveis de homo/transfobia e heterossexismo entre psicólogos, psicoterapeutas e psiquiatras portugueses, também Gila Acker (2017), Cathy Berkman e Gail Zinberg (1997) e Emily Lusenhop (2013) apresentaram evidências que os assistentes 
sociais não se sentem totalmente confortáveis ao lidar com a população LGBTI, revelando, por vezes, atitudes negativas/fóbicas e dificuldades de conciliarem os valores profissionais com os valores pessoais, construídos por influência de convicções sociais, culturais, políticas e religiosas, promotoras da cis/heteronormatividade. Este comportamento não só impacta grandemente na qualidade e sensibilidade das práticas de serviço social, como se reflete na satisfação e avaliação dos utentes que, ao não receberem um serviço competente, tendem a observar os profissionais como "ignorantes" (Jeyasingham, 2008) e desqualificados para os atender.

Esta situação faz, pois, suscitar dúvidas sobre a forma como as escolas de serviço social estão, na verdade, a preparar os alunos para prestarem serviços competentes. A qualidade dos serviços requer, necessariamente, que os profissionais sejam detentores de conhecimentos e capacidades específicas (Dominelli, 1998), bem como competências de tomada de posição sociopolítica (Baines, 2007; Okitikpi \& Aymer, 2010). Sobre este aspeto, Lena Dominelli (1998) acredita que os assistentes sociais "devem saber o que estão a fazer e por que adotam uma determinada ação em vez de outra" (p. 9), sendo que, para tal, a autoavaliação das suas práticas profissionais torna-se fundamental para serem qualitativamente melhoradas.

Dado que, muito provavelmente, a grande parte dos assistentes sociais deparar-seá, na vida laboral (independentemente do seu campo de atuação), com indivíduos com identidades de género e/ou orientações sexuais não normativas, é importante que as escolas de serviço social desenvolvam e providenciem, no contexto da valorização da diversidade e dos direitos humanos, oportunidades de treino e formação sobre género e sexualidade (Berkman e Zinberg, 1997; McPhail, 2008 e Mallon, 1999a,c) - firmando um serviço social queer (Hillock e Mulé, 2016) - a fim de munirem os assistentes sociais de maior confiança e aptidão para comunicarem e intervirem eficazmente com populações LGBTI; prestarem serviços qualificados e não discriminatórios; e desafiarem as desigualdades exigidas pelo código deontológico.

Ajudar os profissionais a reconhecer que a diversidade de género e sexual tem existido em diferentes épocas e lugares de todo o mundo; favorecer a tomada de consciência do grau de homo/transfobia e heterossexismo por via da exploração das suas crenças individuais; fomentar a conciliação de tensões entre valores pessoais e profissionais; promover o contacto, a interação e o diálogo com pessoas com identidades de género e sexualidades não normativas para tomarem conhecimento das suas experiências de vida e necessidades; combater mitos e estereótipos negativos associados 
a elas, disponibilizando informação correta e fidedigna; fomentar a elaboração de linguagens apropriadas, inclusivas e não tendenciosas que orientem a prática profissional; e discutir formas de atuação culturalmente competentes, devem ter lugar nos currículos escolares dos assistentes sociais (Bergh e Crisp, 2004; Erich et al., 2007; Mallon, 1999a; Ramalho, 2015). Presentemente, há uma grande variedade de materiais (American Counseling Association, 2010; Austin et al., 2016; Craig et al., 2016; Equality and Human Rights Commission, 2010; Holman e Goldberg, 2006; Morrow, 2004) que fornecem diretrizes, recomendações e informações práticas para se intervir na diversidade de género e sexual, os quais poderão certamente auxiliar nesse trabalho pedagógico.

\section{Recomendações para a Intervenção na Diversidade de Género e Sexual}

A adoção de práticas anti discriminatórias e anti opressivas exige que os assistentes sociais dominem profundamente os fundamentos sobre os quais o género e a sexualidade foram produzidos e regulados uma vez que a eliminação da opressão implica desafiar a heteromormatividade e o binarismo de género (Burdge, 2007; Markman, 2011; McPhail, 2004), ainda que a situação possa gerar "tensão" e "conflito" resultante das próprias crenças e suposições sobre a organização da realidade social. De facto, não se pode acabar com a opressão de género e sexual se se continuar a ignorar a opressão decorrente da construção (e normalização) das categorias de género e sexuais e da sua hierarquização (McPhail, 2004). Optar pela sua não "desnaturalização" é, em si mesmo, negar a pluralidade de "sexos", "géneros" e "sexualidades" e, consequentemente, reforçar o silenciamento e a exclusão de todas as identidades que não produzem conformidade social.

Todavia, além dos conhecimentos sobre género e sexualidade, os assistentes sociais deverão desenvolver competências e práticas afirmativas na intervenção com populações LGBTI (Ramalho, 2015, p. 132-137). Desde logo, deverá haver uma adequação dos serviços sociais com o objetivo de se transformarem em espaços LGBT-friendly, ambientes verdadeiramente seguros e inclusivos para as pessoas LGBTI. De modo a facilitarem o suporte social, torna-se uma prioridade a eliminação de barreiras que as afastam dos serviços sociais. Além disso, deverá também ser dada particular atenção à relação comunicacional entre assistente social e utente na medida em que esta deverá ser percebida como sendo de abertura, aceitação e confiança, só possível por via do desenvolvimento da empatia, cuja capacidade vai para além do "calçar os sapatos do outro", como refere Dominelli (1998, p. 10). Segundo a autora, requer o seu uso por 
tempo determinado a fim de ser desenvolvida uma "profunda compreensão" dos sentimentos e emoções e, ao mesmo tempo, ajudar na reflexão sobre a natureza de "privilegiado" do próprio. Por forma a que o atendimento seja acolhedor e livre de discriminação os profissionais deverão evitar assumir, à partida, que todos os indivíduos que recorrem até si têm uma identidade de género ou orientação sexual normativa. Tal suposição, quando expressa em comportamentos, poderá ser geradora de desconforto e mal-estar (Bergh \& Crisp, 2004; Charnley \& Langley, 2007). A questão do tratamento das pessoas com diversidade de género é, em si mesma, bastante sensível porque poderá ser constrangedor e, até, ofensivo fazer uso do nome com o qual elas não reconhecem a sua identidade. Deste modo, deverá ser-lhes perguntado como desejam ser tratadas, empregar esse nome nas interações e registá-lo em campo apropriado para que, em atendimentos futuros, sejam tratadas e respeitadas convenientemente. É importante, também, que os formulários de admissão e de atendimento social possam dar expressão à diversidade de género e sexual. Por isso, deixar um espaço em branco para que os utentes se autoidentifiquem poderá ser, por exemplo, uma prática a adotar dado que não reforça o binarismo nem ignora as sexualidades não normativas. A mesma situação se aplica ao nível da investigação quantitativa, sendo importante que os inquéritos por questionário passem a integrar opções de género que extrapolem o binómio masculino/feminino dado que ele tende a homogeneizar os participantes dos estudos e, com isso, os próprios resultados, mascarando a compreensão dos fenómenos sociais.

Garantir que os serviços sociais sejam espaços seguros para as pessoas LGBTI passa, também, por promover junto da equipa de trabalho uma política de "tolerância zero" perante o comportamento discriminatório (Bergh \& Crisp, 2004; Mallon, 1999c). Deste modo, deverá ser assegurado que todos os profissionais (sobretudo os que se encontram no primeiro contacto) possuem competências adequadas para lidar com a diversidade de género e sexual e oferecer um tratamento respeitoso. Somente através de mudanças culturais organizacionais intencionais e deliberadas é que poderão ser desenvolvidos ambientes favoráveis onde as pessoas LGBTI se sintam bem-vindas, acolhidas e com as suas necessidades atendidas.

Reconhecendo, ainda, que o ambiente externo aos serviços sociais poderá ser ativamente hostil para grande parte das pessoas LGBTI, é também importante que os assistentes sociais, ao estarem comprometidos com a eliminação da opressão, desenvolvam trabalho de advocacia (Mallon, 1999c, p. 140) para alterarem essa realidade. Deverão, pois, envolver-se na afirmação das minorias de género e sexuais em diferentes 
contextos profissionais (como reuniões de trabalho ou conferências, por exemplo), assim como na educação social, cívica e política por via do desenvolvimento de programas de sensibilização e formação, o mais descentralizados possíveis em termos geográficos, e que abranjam tanto instituições públicas como privadas, com o objetivo de dotar a população de maiores conhecimentos em matéria de género e sexualidade. Para isso, poderá contribuir a produção e disseminação de materiais educativos (livros, manuais, sites, jogos, etc.) e recursos performativos (teatros, filmes) que ajudem a desafiar os preconceitos e a estimular uma maior abertura, respeito e aceitação das pessoas LGBTI. A família, a escola e as organizações comunitárias são agentes vitais a serem envolvidos nesta intervenção porque o tipo de respostas que produzem (positivas ou negativas) poderá transformá-las em agentes protetores ou de risco psicossocial para muitas pessoas LGBTI. É, pois, tarefa dos profissionais de serviço social ajudá-las a compreender que as crenças que possuem sobre a masculinidade, a feminilidade e a orientação sexual podem afetar as suas decisões, assim como ensiná-las sobre as múltiplas vulnerabilidades e opressões que pessoas LGBTI são alvo e possibilitar-lhes a aquisição de informações e competências para, em vez de rejeição, providenciem suporte e aceitação (Cohen et al., 2006).

Por fim, mas sem esgotar as recomendações, considera-se que a intervenção dos assistentes sociais também deverá contemplar a (re)ativação das redes formais e informais das pessoas LGBTI. Esta ação, focada na criação e/ou restauração de vínculos com a esfera social, poderá ser facilitada pelo estabelecimento de alianças com alguns movimentos sociais, organizações de defesa de direitos civis e outras organizações profissionais. Fomentar a conexão e diminuir a distância física e interpessoal poderá ser uma estratégia para evitar o seu isolamento e o agravamento das vulnerabilidades e, em simultâneo, promover o cumprimento dos seus direitos humanos.

\section{Conclusão}

O presente artigo deu conta que, apesar das exigências deontológicas e dos compromissos assumidos pelas organizações nacionais e internacionais de serviço social relacionados com a necessidade de proteção e preservação dos direitos das pessoas LGBTI, os assistentes sociais continuam a apresentar parcos conhecimentos e competências para intervir adequadamente com populações com diversidade de género e sexual, em boa parte porque as escolas de serviço social tendem a fornecer pouca formação sobre género e sexualidade. Neste sentido, espera-se que as mesmas promovam 
um esforço de incluir as temáticas do género e da sexualidade nos seus planos de estudo e providenciem, com maior intensidade, oportunidades de formação sobre a população LGBTI com vista ao desenvolvimento de competências específicas por parte de futuros assistentes sociais.

\section{Referências bibliográficas:}

Acker, G. (2017). Transphobia among students majoring in the helping professions. Journal of Homosexuality, O(0), 1-19.

American Counseling Association (2010). Competencies for counseling with transgender clients. Journal of LGBT Issues in Counseling, 4(3-4), 135-159.

Associação dos Profissionais de Serviço Social (2018). Código deontológico dos assistentes sociais em Portugal. Lisboa: APSS.

Austin, A., Craig, S., Alessi, E., Wagaman, A., Paceley, M., Dziengel, L., \& Balestrery, J. (2016). Guidelines for transgender and gender nonconforming (TGNC) affirmative education: Enhancing the climate for TGNC students, staff and faculty in social work education. Alexandria, VA: Council on Social Work Education.

Baines, D. (2007). Doing anti-oppressive practice: Building transformative politicized social work. Black Point, Nova Scotia: Fernwood Publishing.

Barroco, M. L. (2003). Ética, direitos humanos e diversidade. Revista Presença Ética, 3. Disponível em https://presenetica.wordpress.com/

Bergh, N., \& Crisp, C. (2004). Defining culturally competence practice with sexual minorities: Implications for social work education and practice. Journal of Social Work Education, 40(2), 221-238.

Berkman, C., \& Zinberg, G. (1997). Homophobia and heterosexism in social workers. Social Work, 42, 319-332.

Brown, H., \& Cocker, C. (2011). Social work values and ethical practice: Moving beyond anti-discriminatory or anti-oppressive practice. Em H. Brown \& C. Cocker (Orgs.), Social work with lesbians \& gay men (pp. 97-115). Londres: Sage.

Burdge, B. (2007). Bending gender, ending gender: Theoretical foundations for social work practice with the transgender community. Social Work, 52, 243-250.

Charnley, H., \& Langley, J. (2007). Developing cultural competence as a framework for anti-heterosexist social work practice: Reflection from the UK. Journal of Social Work, 7(3), 207-321.

Cohen, H., Padilla, Y., \& Aravena, V. (2006). Psychosocial support for families of gay, lesbian, bisexual, and transgender people. Em D. Morrow \& L. Messinger (Orgs.), Sexual orientation and gender expression in social work practice: Working with gay, lesbian, bisexual, and transgender people (pp. 153-173). Nova Iorque: Columbia University Press.

Craig, S. L., Alessi, E. J., Fisher-Borne, M., Dentato, M. P., Austin, A., Paceley, M., Wagaman, A., Arguello, T., Lewis, T., Balestrery, J. E., \& Van Der Horn, R. (2016). Guidelines for affirmative social work education: Enhancing the climate for LGBQQ 
students, staff, and faculty in social work education. Alexandria, VA: Council on Social Work Education.

Dominelli, L. (1998). Anti-oppressive practice in context. Em R. Adams, L. Dominelli \& M. Payne (Orgs.), Social work: Themes, issues and critical debates (pp. 322). Londres: Macmillan Press.

Equality and Human Rights Commission (2010). Provision of goods, facilities and services to trans people. Guidance for public authorities: Meeting your equality duties and human rights obligations. Manchester, Glasgow \& Cardiff: Equality and Human Rights Commission.

Erich, S., Boutte'-Queen, N., Donnelly, S., \& Tittsworth, J. (2007). Social work education: Implications for working with the transgender community. Journal of Baccalaureate Social Work, 12(2), 42-52.

Hicks, S. (2008). Thinking through sexuality. Journal of Social Work, 8(1), 65-82.

Hillock, S., \& Mulé, N. (Orgs.) (2016). Queering social work education. Vancouver: University of British Columbia Press.

Holman, C., \& Goldberg, J. (2006). Social and medical advocacy with transgender people and loved ones: Recommendations for BC clinicians. Vancouver: Vancouver Coastal Health Transgender Health, Transcend \& Canadian Rainbow Health Coalition.

International Federation of Social Workers (2014). Sexual orientation and gender expression. Disponível em http://ifsw.org/policies/sexual-orientation-and-genderexpression/

Jeyasingham, D. (2008). Knowledge/ignorance and the construction of sexuality in social work education. Social Work Education, 27(2), 138-151.

Lusenhop, E. (2013). The impact of homophobia and transphobia on the professional development of LGBT social workers during graduate school. Northampton, Dissertação de mestrado em serviço social apresentada à Smith College School for Social Work.

Mackelprang, R., Ray, J., \& Hernandez-Peck, M. (1996). Social work education and sexual orientation: Faculty, student, and curriculum issues. Journal of Gay \& Lesbian Social Services, 5(4), 17-31.

Mallon, G. (1999a). Knowledge for practice with transgendered persons. Journal of Gay and Lesbian Social Services, 10(3/4), 1-18.

Mallon, G. (1999b). Gay and lesbian adolescents and their families. Journal of Gay \& Lesbian Social Services, 10(2), 69-88.

Mallon, G. (1999c). A call for organizational trans-formation. Journal of Gay \& Lesbian Social Services, 10(2), 131-142.

Markman, E. (2011). Gender identity disorder, the gender binary, and transgender oppression: Implications for ethical social work. Smith College Studies in Social Work, 81(4), 314-327.

McPhail, B. (2008). Re-gendering the social work curriculum: New realities and complexities. Journal of Social Work Education, 44, 33-52.

Messinger, L. (2004). Out in the field: Gay and lesbian social work students' experiences in field placement. Journal of Social Work Education, 40, 187-204. 
Moita, M. G. (2001). Discursos sobre a homossexualidade no contexto clínico: A homossexualidade de dois lados do espelho. Porto, Tese de doutoramento em ciências biomédicas apresentada ao Instituto de Ciências Biomédicas de Abel Salazar da Universidade do Porto.

Morrow, D. (2004). Social work practice with gay, lesbian, bisexual, and transgender adolescents. Families in Society: The Journal of Contemporary Social Services, 85(1), 91-99.

Morton, J., Jeyasingham, D., \& Hicks, S. (2013). The social work of sexuality: Rethinking approaches to social work education. The Higher Education Academy, 2(2), $1-4$.

Newman, P., Bogo, M., \& Daley, A. (2009). Breaking the silence: Sexual orientation in social work field education. Journal of Social Work Education, 45, 7-27.

Okitikpi, T., \& Aymer, C. (2010). Key concepts in anti-discriminatory social work. Londres: Sage.

Pyne, J. (2016). Queer and trans collisions in the classroom: A call to throw open theoretical doors in social work education. Em S. Hillock \& N. Mule (Orgs.), Queering social work education (pp.54-72). Vancouver: University of British Columbia Press.

Radecki, K.(2011). Sexuality and social work: Prevalence of human sexuality in MSW curriculum. Sacramento, Dissertação de mestrado em serviço social apresentado à California State University.

Ramalho, N. (2015). Competências e práticas afirmativas dos assistentes sociais com famílias e pessoas lésbicas, gays, bissexuais e transgénero. Em M. I. Carvalho (Org.), Serviço Social com Famílias (pp. 125-140). Lisboa: Pactor.

Rocha, A. (2009). Sexualidade e diversidade sexual na formação em serviço social nos cursos de $1^{\circ}$ ciclo no Instituto Superior Miguel Torga e na Faculdade de Psicologia e Ciências da Educação da Universidade de Coimbra. Coimbra, Dissertação de mestrado em serviço social apresentada ao Instituto Superior Miguel Torga. 